\title{
Design and Fabrication of Nanoscale IDTs Using Electron Beam Technology for High-Frequency SAW Devices
}

\author{
Wei-Che Shih, ${ }^{1}$ Ying-Chung Chen, ${ }^{1}$ Wei-Tsai Chang, ${ }^{1}$ Chien-Chuan Cheng, ${ }^{2}$ \\ Pei-Chun Liao, ${ }^{1}$ and Kuo-Sheng Kao ${ }^{3}$ \\ ${ }^{1}$ Department of Electrical Engineering, National Sun Yat-sen University, No. 70 Lienhai Road, Kaohsiung 80424, Taiwan \\ ${ }^{2}$ Department of Electronic Engineering, De Lin Institute of Technology, Lane 380, No. 1 Qingyun Road, Tucheng District, \\ New Taipei 23654, Taiwan \\ ${ }^{3}$ Department of Computer and Communication, Shu-Te University, No. 59 Hengshan Road, Yanchao District, \\ Kaohsiung 82445, Taiwan
}

Correspondence should be addressed to Kuo-Sheng Kao; kks@stu.edu.tw

Received 13 December 2013; Accepted 21 February 2014; Published 7 April 2014

Academic Editor: Liang-Wen Ji

Copyright (C) 2014 Wei-Che Shih et al. This is an open access article distributed under the Creative Commons Attribution License, which permits unrestricted use, distribution, and reproduction in any medium, provided the original work is properly cited.

High-frequency Rayleigh-mode surface acoustic wave (SAW) devices were fabricated for $4 \mathrm{G}$ mobile telecommunications. The RF magnetron sputtering method was adopted to grow piezoelectric aluminum nitride (AlN) thin films on the $\mathrm{Si}_{3} \mathrm{~N}_{4} / \mathrm{Si}$ substrates. The influence of sputtering parameters on the crystalline characteristics of AlN thin films was investigated. The interdigital transducer electrodes (IDTs) of aluminum (Al) were then fabricated onto the AlN surfaces by using the electron beam (e-beam) direct write lithography method to form the $\mathrm{Al} / \mathrm{AlN} / \mathrm{Si}_{3} \mathrm{~N}_{4} / \mathrm{Si}$ structured SAW devices. The Al electrodes were adopted owing to its low resistivity, low cost, and low density of the material. For $4 \mathrm{G}$ applications in mobile telecommunications, the line widths of $937 \mathrm{~nm}, 750 \mathrm{~nm}, 562 \mathrm{~nm}$, and $375 \mathrm{~nm}$ of IDTs were designed. Preferred orientation and crystalline properties of AlN thin films were determined by X-ray diffraction using a Siemens XRD-8 with $\mathrm{CuK} \alpha$ radiation. Additionally, the cross-sectional images of AlN thin films were obtained by scanning electron microscope. Finally, the frequency responses of high-frequency SAW devices were measured using the E5071C network analyzer. The center frequencies of the high-frequency Rayleigh-mode SAW devices of $1.36 \mathrm{GHz}, 1.81 \mathrm{GHz}, 2.37 \mathrm{GHz}$, and $3.74 \mathrm{GHz}$ are obtained. This study demonstrates that the proposed processing method significantly contributes to high-frequency SAW devices for wireless communications.

\section{Introduction}

After the first commercial cell phone (Motorola DynaTAC) was designed and fabricated by Martin Cooper in 1983 [1], various acoustic wave devices are investigated for use in mobile telecommunications, such as a surface acoustic wave (SAW) oscillator, thin film bulk acoustic resonator (TFBAR), and high-tone bulk acoustic resonator (HBAR) [2-4]. These devices are also applicable in popular video categories, such as PDAs, smartphones, and connected music players. In the meantime, frequency bandwidth of communication systems has received considerable attention in recent years [5]. Therefore, high-frequency SAW devices have been widely studied, owing to their low cost, small size, lightweight nature, simple structure, good reproducibility, and diversity of functional applications.

Piezoelectric films such as lead zirconate titanate (PZT), zinc oxide $(\mathrm{ZnO})$, and aluminum nitride (AlN) thin films have been applied in acoustic devices for various applications [6-15]. In this study, the piezoelectric thin films of AlN are adopted for the applications of high-frequency SAW devices, owing to its better quality factor, high acoustic velocity, and high electromechanical coupling coefficient. The global systems for mobile communications can be classified into several systems, including GSM-900, DCS-1800, PCS-1900, WCDMA, and LTE. To promote the occupation efficiency of bandwidth segment, several studies have attempted to design and fabricate high-frequency acoustic devices for 
telecommunications [16-19]. In this study, a novel procedure with the electron beam (e-beam) direct write lithography method to design and fabricate high-frequency Rayleighmode SAW devices was proposed.

This study attempts to optimize the fabrication processes by examining the influence of sputtering parameters on the performance of high-frequency AlN-based SAW devices. In particular, the effects of e-beam direct write lithography on the characteristics of the surfaces of AlN thin films are investigated. Given the continuous scaling down of the modern fabrication processes in line widths, conventional SAW devices are reaching their physical limits and alternative technologies are needed. The need for this requirement can be revealed when a specific liftoff method is adopted to realize the nanoscale interdigital transducer electrodes (IDTs). In this study, nanoscale IDTs are fabricated by using two patterning methods; those are conventional photolithography method combined with wet-etching and the e-beam direct write lithography method combined with dryetching. Finally, the acoustic performances of high-frequency Rayleigh-mode SAW devices are studied using a network analyzer system.

\section{Experimental}

In this study, low-stress silicon nitride was deposited on the silicon substrates by low-pressure chemical vapor deposition (LPCVD) as the supporting layer for the SAW devices. After the substrates were cleaned using a normal process, $c$-axis orientation AlN thin films were deposited using reactive RF magnetron sputtering. The sputtering system was evacuated to a base pressure of $5 \times 10^{-7}$ torr by using a diffusion pump to achieve the required deposition conditions. Table 1 details sputtering conditions to prepare AlN thin films with a caxis orientation. An attempt was also carried out to obtain the optimal IDTs patterns by using two kinds of patterning methods.

The first method adopts the conventional photolithography method with wet-etching. The IDTs are patterned by the photolithography using photomask as shown in Figure 1. The Al thin films are then deposited by a DC sputtering system. Table 2 details the sputtering conditions to prepare Al thin films. The IDTs are achieved using the liftoff method as shown in Figure 2. The second method for fabricating IDTs involves combining e-beam direct write with dry-etching. The accelerated voltage of an e-beam writer is set at $50 \mathrm{kV}$, and the current is controlled at $100 \mathrm{pA}$. After the electron resistance is coated on $\mathrm{Al}$ thin films, the e-beam writer directly writes to define the IDTs according to the pattern shown in Figure 3. The IDTs are then achieved with inductance coupling plasma (ICP) dry-etching process to remove unneeded $\mathrm{Al}$ thin films. Figure 4 shows the fabrication steps. Table 3 lists the designed parameters of IDTs for the SAW devices.

The preferred orientation and crystalline properties of the AlN thin films are determined by X-ray diffraction scanning between $20^{\circ}$ and $60^{\circ}$ at the speed of $0.05^{\circ}$ per second using a Siemens D8 with $\mathrm{CuK} \alpha$ radiation. The surface morphologies and composition distribution of thin films are observed by
TABLE 1: Deposition parameters of AlN thin films.

\begin{tabular}{ll}
\hline Target & $\mathrm{Al}(99.995 \%)$ \\
Substrate-to-target distance $(\mathrm{mm})$ & 50 \\
Base pressure (Torr) & $5 \times 10^{-7}$ \\
Substrate temperature $\left({ }^{\circ} \mathrm{C}\right)$ & 300 \\
$\mathrm{RF}$ power $(\mathrm{W})$ & $200,250,300$ \\
Sputtering pressure (mTorr) & $5,10,15$ \\
$\mathrm{~N}_{2} /\left(\mathrm{N}_{2}+\right.$ Ar) & $60 \%$ \\
Time durations (hours) & 3 \\
Deposition rate & $667 \mathrm{~nm} / \mathrm{hour}$ \\
\hline
\end{tabular}

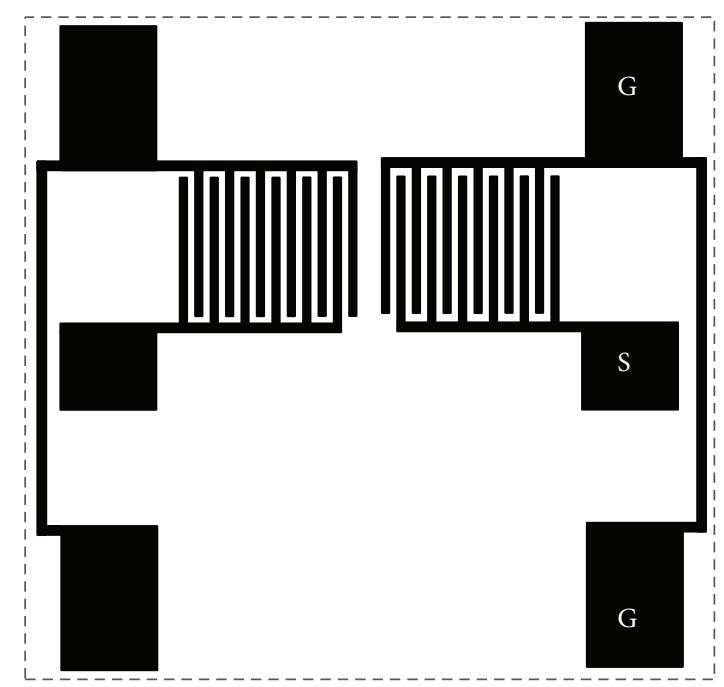

FIGURE 1: The photomask of conventional photolithography.

TABLE 2: Deposition parameters of Al thin films.

\begin{tabular}{ll}
\hline Target & $\mathrm{Al}(99.995 \%)$ \\
Substrate-to-target distance $(\mathrm{mm})$ & 50 \\
Base pressure (Torr) & $1 \times 10^{-6}$ \\
Substrate temperature $\left({ }^{\circ} \mathrm{C}\right)$ & $\mathrm{R} . \mathrm{T}$. \\
DC power $(\mathrm{W})$ & 100 \\
Sputtering pressure (mTorr) & 4 \\
Ar $(\mathrm{sccm})$ & 10 \\
\hline
\end{tabular}

scanning electron microscope (SEM) (JEOL-6700 FESEM) with an energy dispersive spectrometer. The accelerated voltage of SEM is set at $10 \mathrm{kV}$, and the magnification is controlled over a range of magnitude ranging from 3000x to 60000x. Finally, the frequency responses of Rayleigh-mode SAW devices are measured using the network analyzer system (E5071C).

\section{Results and Discussion}

3.1. Structural and Morphological Properties of AlN Thin Films. A strongly $c$-axis orientated and a uniform surface 

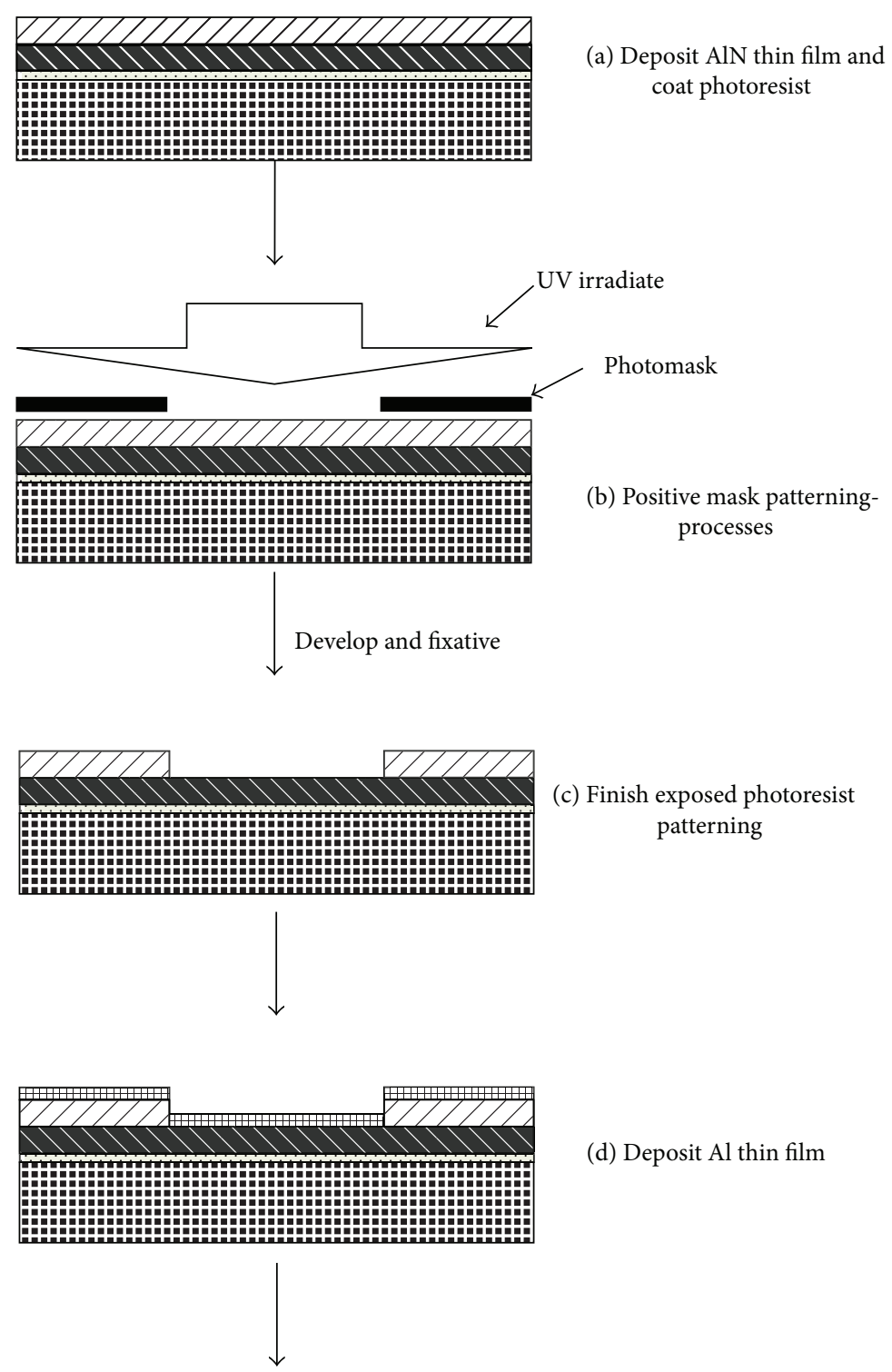

(d) Deposit Al thin film

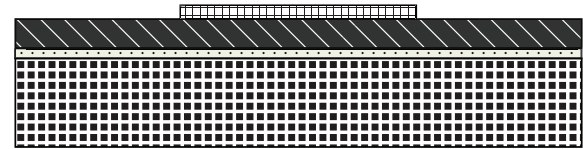

(e) Complete the SAW device by liftoff method

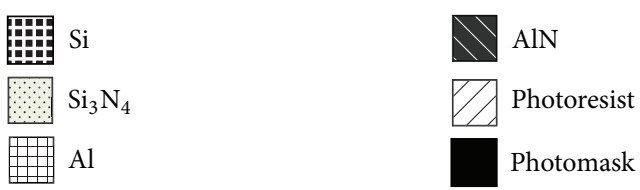

FIGURE 2: The fabrication steps of SAW devices using conventional photolithography method combined with wet-etching.

morphology are the ideal physical properties of piezoelectric films for high-frequency SAW applications. Therefore, this study investigated two major deposition parameters to optimize the characteristics of highly textured AlN thin films, including sputtering pressure and RF power. Firstly, the sputtering pressure was varied and set to $5 \mathrm{mTorr}, 10 \mathrm{mTorr}$, and 15 mTorr to determine the effects of sputtering pressure on the crystalline growth of AlN thin films. The substrate temperature was fixed at $300^{\circ} \mathrm{C}$, owing to that the adequate thermal can improve the piezoelectric properties of AlN 


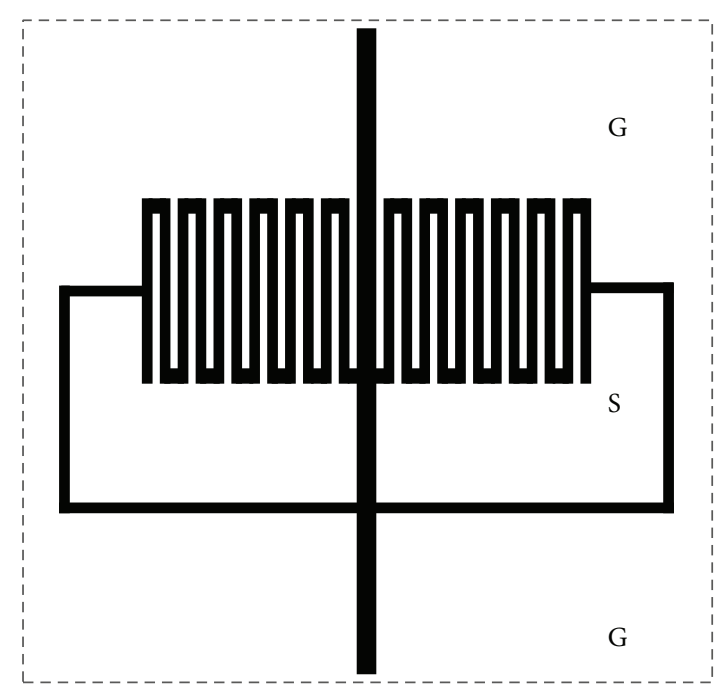

FIGURE 3: The mask of e-beam lithography.

TABLE 3: The designed parameters of IDTs for the Rayleigh-mode SAW devices.

\begin{tabular}{lc}
\hline & E-beam direct write \\
\hline Input IDT electrode (pairs) & 30 \\
Output IDT electrode (pairs) & 30 \\
Area of device $(\mu \mathrm{m})$ & $600 \times 600$ \\
Wavelength $\lambda(\mathrm{nm})$ & $937,750,562,375$ \\
The overlapped length $(\mu \mathrm{m})$ & 60 \\
Delay line $(\mu \mathrm{m})$ & 110 \\
Distance between two electrodes $(\mu \mathrm{m})$ & 150 \\
\hline
\end{tabular}

thin films. According to Figure 5, excellent (002) orientated crystals with a small full width at half maximum (FWHM) can be obtained in samples at sputtering pressure of 10 mTorr. Additionally, as revealed by the surface morphologies and cross-sectional images in Figure 6, the surface exhibited uniform cobblestone-like crystallites, and the columnar textured AlN thin film appeared.

Secondly, the RF power was varied to investigate the influence on the characteristics of AlN thin films. The XRD patterns in Figure 7 display the variations in the $2 \theta$ range of 30 to 60 degrees with the RF power. The results showed that AlN thin films deposited at $250 \mathrm{~W}$ exhibited a sharp (002) peak and small value of FWHM. Figure 8 reveals that the surface roughness of AlN thin films increased with an increasing of RF power. Therefore, the RF power was fixed at $250 \mathrm{~W}$ to achieve a higher electromechanical coupling coefficient and smooth surface shaped AlN thin films.

Finally, AlN thin films with a smooth surface, uniform grain size, and strongly $c$-axis orientated crystallization were deposited based on the optimal substrate temperature of $300^{\circ} \mathrm{C}$, RF power of $250 \mathrm{~W}$, and sputtering pressure of 10 mTorr. The obtained surface morphologies of AlN thin films appeared to be dense with smooth surfaces and strongly textured columnar structures. As is believed, hexagonal
TABLE 4: The different line widths of IDTs fabricated with various e-beam exposure durations.

\begin{tabular}{lc}
\hline Line widths of IDT $(\mathrm{nm})$ & Exposure duration $(\mu \mathrm{s})$ \\
\hline 937 & $2.2 \sim 1.7$ \\
750 & $2.2 \sim 1.85$ \\
562 & $2.3 \sim 2.2$ \\
375 & $2.6 \sim 2.5$ \\
\hline
\end{tabular}

crystals exhibit piezoelectricity of unity with a (002) preferred orientation $[20,21]$.

\subsection{Conventional Photolithography and E-Beam Direct Write} Processes. Figure 9 shows the optical microscopic (OM) images of IDTs by using the conventional photolithography method. Analysis results indicate that the IDTs morphology has several unfavorable properties, including a blurred morphology, damaged IDTs, and the breakage status. These properties are owing to the inability of the nanoscaled width of the photoresist to sustain the side etching of the acetone [22].

Although this study designed four different line widths of IDTs, the conventional photolithography method failed to fabricate IDTs with nanoscale. To resolve the above poor resolution, the e-beam direct write method is adopted.

In this study, high-frequency Rayleigh-mode SAW devices are fabricated using e-beam lithography, owing to its high resolution, high optical-sensitivity, precise alignment, low defect density, and easy preservation ability. Moreover, a less process time is expected due to the less writing area.

The ICP dry-etching process accompanied by the ebeam direct write method will be a promising solution for the nanoscale process. Several line widths of IDTs are designed and tested in advance to determine the limitations of the e-beam direct writer and the withstanding ability of 


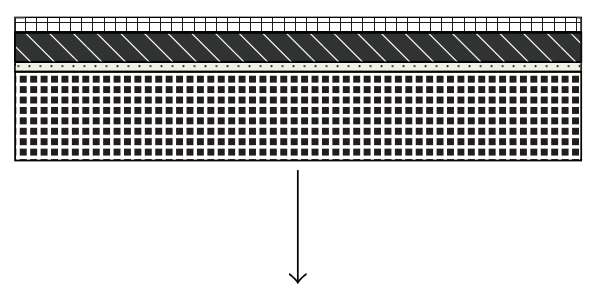

(a) Deposit AlN and Al thin films

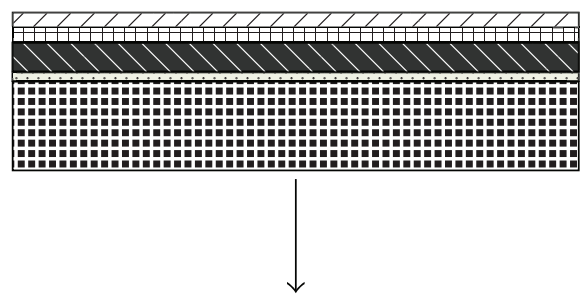

(b) Coat electron resistance on $\mathrm{Al}$ thin films

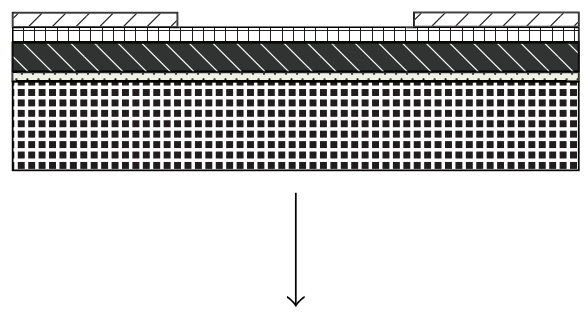

(c) E-beam direct write to define IDT patterns

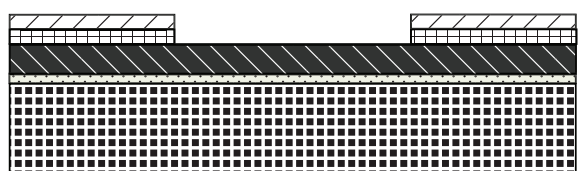

(d) Unneeded Al thin films were removed using ICP-etching method

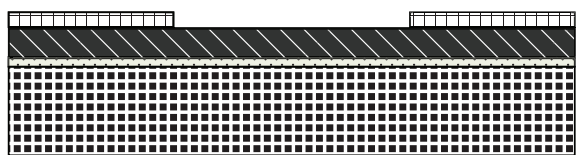

(e) Complete the SAW device

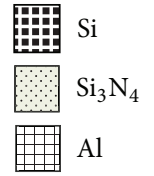

AlN

Electron resistance

Figure 4: The fabrication steps of SAW devices using e-beam direct write combined with dry-etching.

the electron resistance for plasma etching. Notably, the exposure time must be adjusted because the exposure duration of e-beam is a dominant parameter. The insufficient exposures reveal an unclear line width and bad resolution, as shown in Figure 10(a). Conversely, the overdose exposures caused excessive irradiation and damaged the line widths.
Figure 10(b) shows the clear line widths with suitable exposures controlled. Table 4 lists the durations of controlled exposures. Figure 11 shows the OM images of IDTs after the ICP-etching process. Analysis results indicate that the e-beam direct write method exhibits several favorable advantages, including an obvious morphology, acceptable processing 


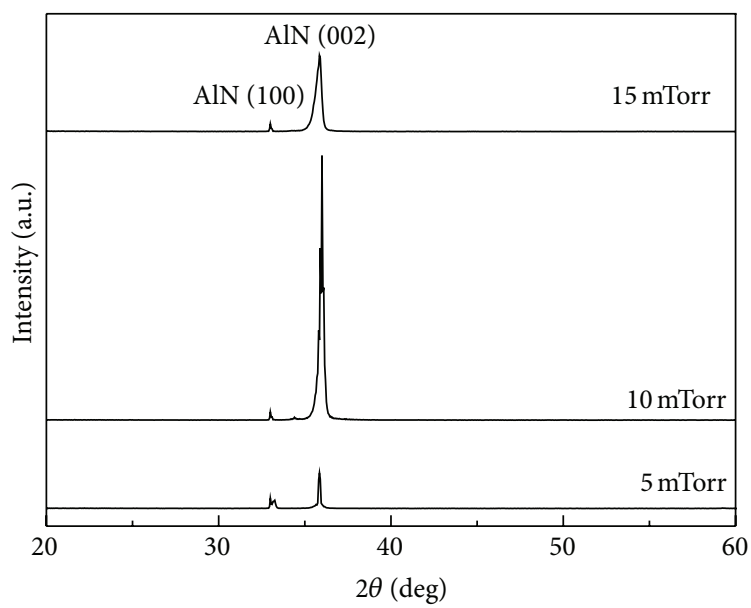

Figure 5: The $\theta-2 \theta$ X-ray scans of the AlN thin films deposited with various sputtering pressures.

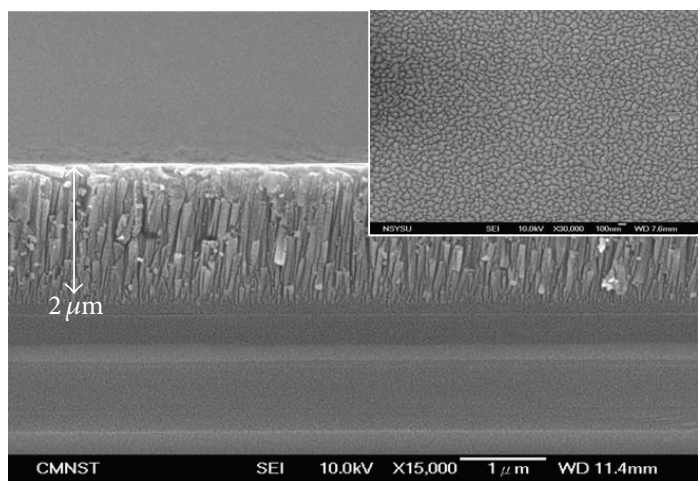

FIGURE 6: The surface morphology and cross-sectional image of the AlN thin films deposited at 10 mTorr.

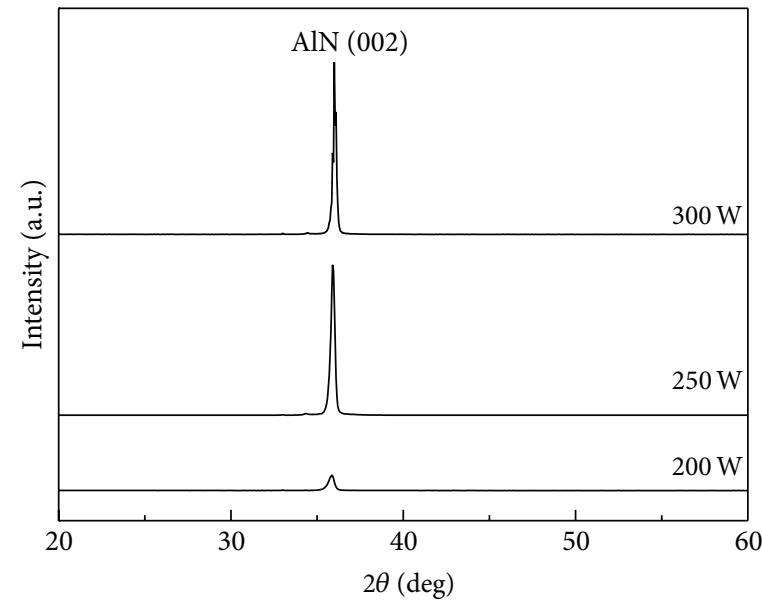

Figure 7: The $\theta-2 \theta$ X-ray scans of the AlN thin films deposited with various RF powers.

time, high yield, and large electrodes area for electrical connections. Therefore, in this study, IDTs with nanoscale widths are obtained using the e-beam direct write method.

\subsection{Frequency Responses of High-Frequency Rayleigh-Mode} $S A W$ Devices. Figure 12 shows the frequency responses measured using the E5071C network analyzer for the SAW devices with various line widths patterns of IDTs. The center frequencies of the high-frequency Rayleigh-mode SAW devices of $1.36 \mathrm{GHz}, 1.81 \mathrm{GHz}, 2.37 \mathrm{GHz}$, and $3.74 \mathrm{GHz}$ are obtained for the line widths of $937 \mathrm{~nm}, 750 \mathrm{~nm}, 562 \mathrm{~nm}$, and $375 \mathrm{~nm}$ of IDTs, respectively. The measured center frequencies of all SAW devices are close to the theoretical calculations. The results demonstrate that the proposed processing method 


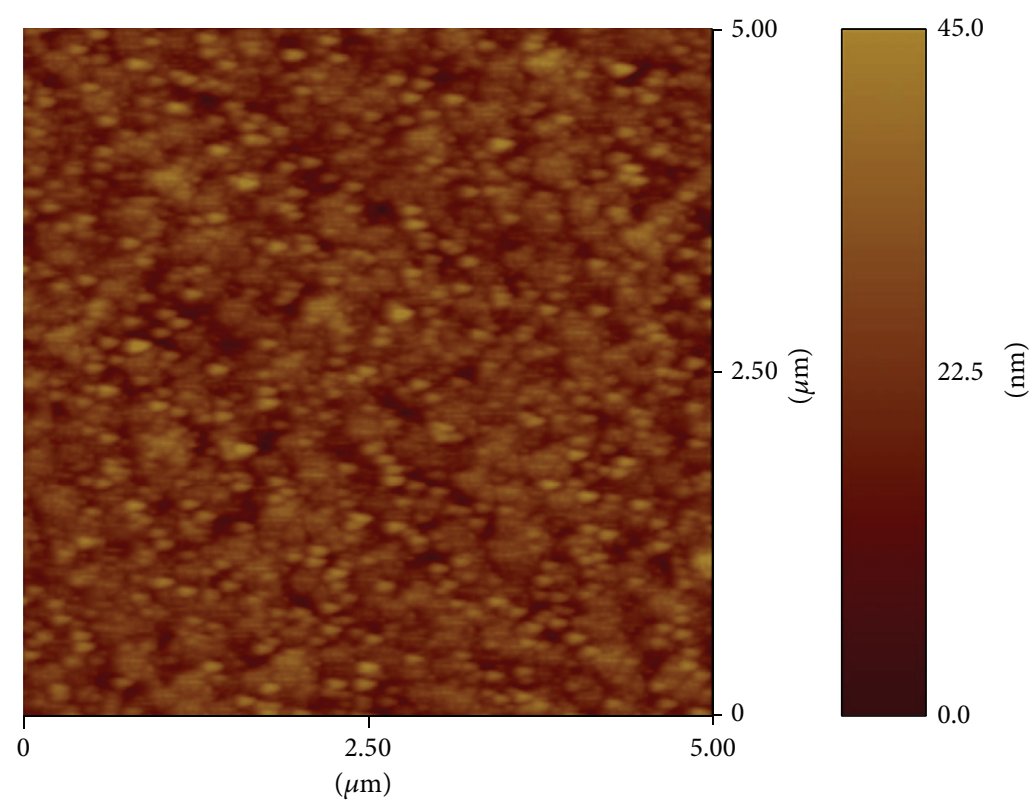

$\begin{array}{lll}\text { Image statistics } & & \text { Box statistics } \\ \text { Image } R_{\mathrm{me}}\left(R_{q}\right) & 6.248 \mathrm{~nm} & R_{\mathrm{ms}}\left(R_{q}\right) \\ \text { Image } R_{a} & 4.953 \mathrm{~nm} & \text { Mean roughness }\left(R_{a}\right) \\ \text { Image } R_{\max } & 49.551 \mathrm{~nm} & \end{array}$

(a)

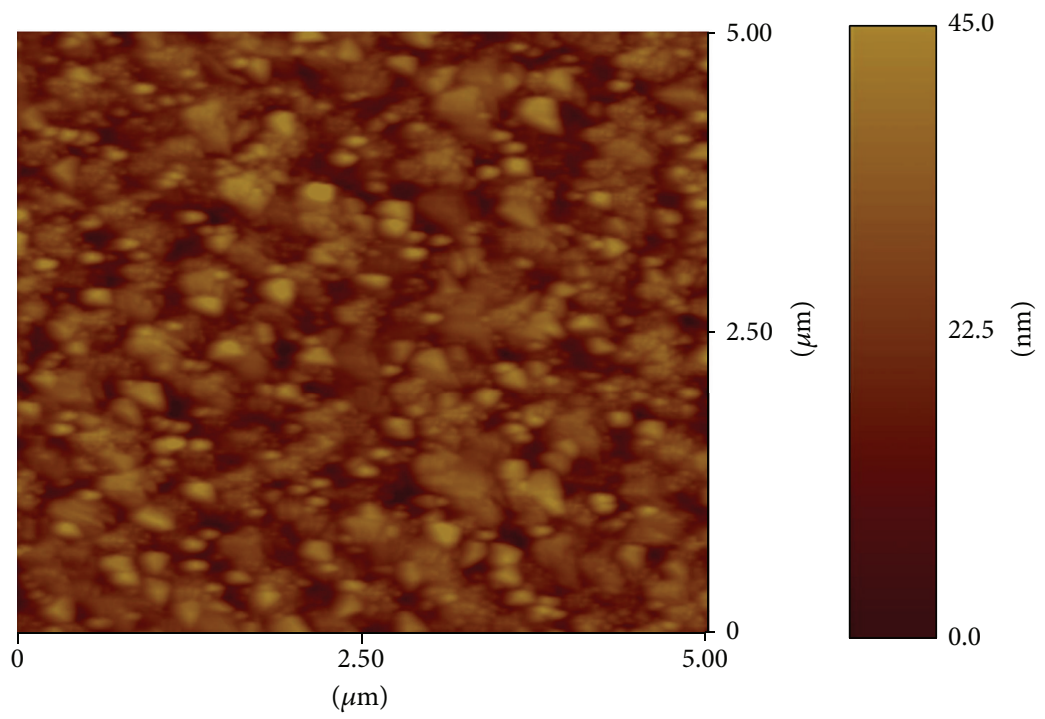

$\begin{array}{lll}\text { Image statistics } & & \text { Box statistics } \\ \text { Image } R_{\mathrm{me}}\left(R_{q}\right) & 8.047 \mathrm{~nm} & R_{\mathrm{ms}}\left(R_{q}\right) \\ \text { Image } R_{a} & 6.437 \mathrm{~nm} & \text { Mean roughness }\left(R_{a}\right) \\ \text { Image } R_{\max } & 62.392 \mathrm{~nm} & \end{array}$

(b)

FIGURE 8: The atomic force microscopy images of AlN thin films deposited with RF powers of (a) $250 \mathrm{~W}$ and (b) $300 \mathrm{~W}$. 


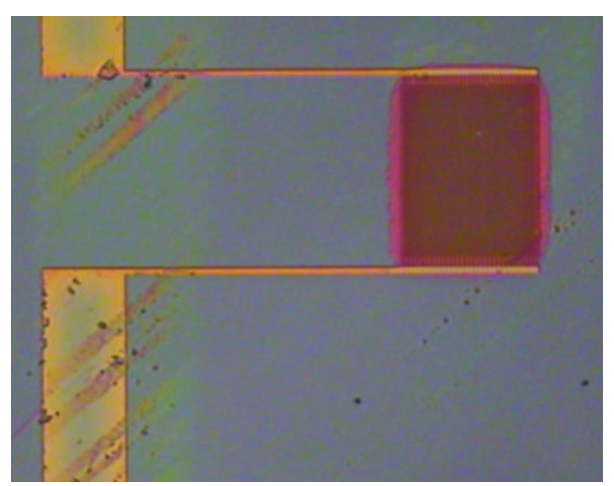

FIGURE 9: The OM images of IDTs after the conventional liftoff method.

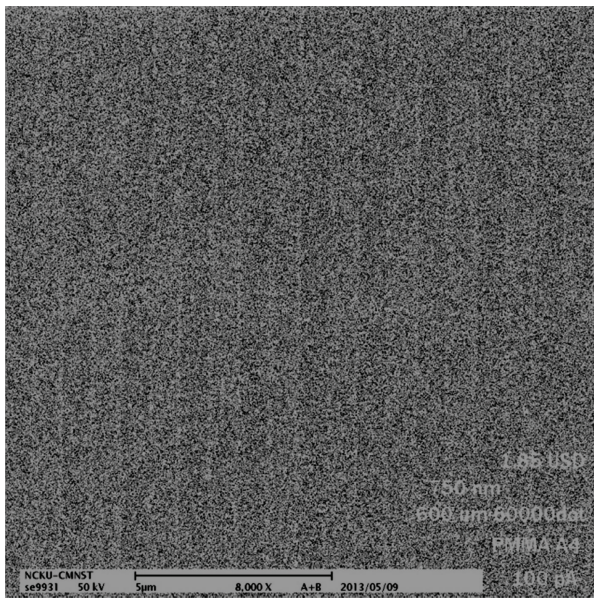

(a)

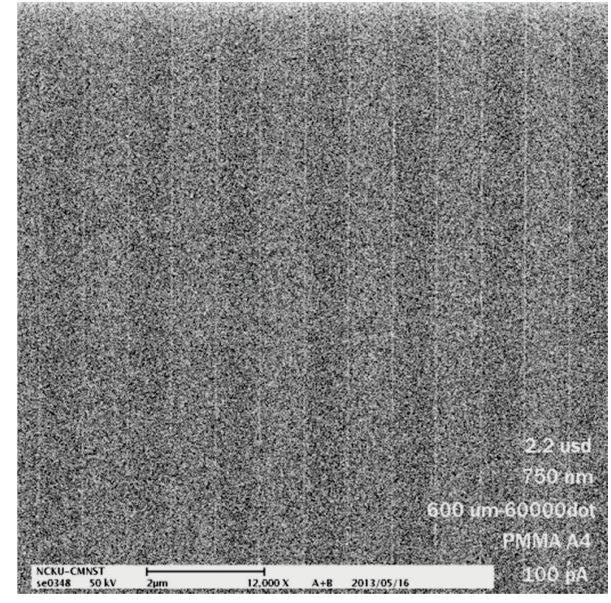

(b)

FIGURE 10: The exposure duration of e-beam: (a) insufficient exposures and (b) suitable exposures.

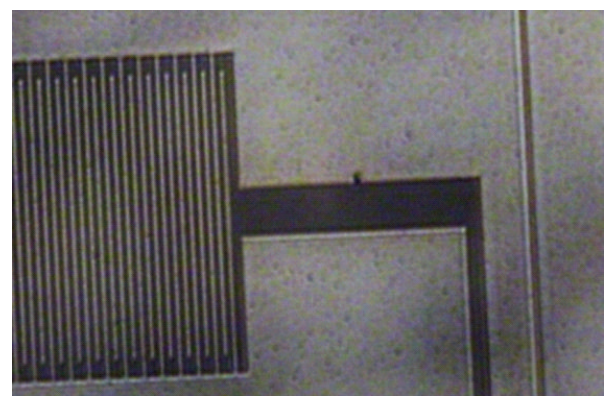

FIgURE 11: The OM images of IDTs after the e-beam direct write and ICP-etching.

significantly contributes to high-frequency SAW devices for wireless communications.

\section{Conclusions}

This study describes the fabrication of a high-frequency Rayleigh-mode SAW device for $4 \mathrm{G}$ mobile telecommunication application. AlN thin films are deposited on $\mathrm{Si}_{3} \mathrm{~N}_{4} / \mathrm{Si}$ substrates by reactive RF magnetron sputtering. The SAW devices are fabricated using the e-beam lithography method. The AlN thin films exhibit excellent properties, including (002) preferred orientation, smooth surface, uniform grain, and columnar structure with suitable sputtering parameters controlled. Additionally, e-beam direct write methods combined with ICP-etching methods are adopted to develop the nanoscaled IDTs. The proposed method has several advantages, including high density plasma, uniform etched surfaces, and high etching rate, making it feasible for fabricating the nanoscaled devices. Finally, the SAW devices 


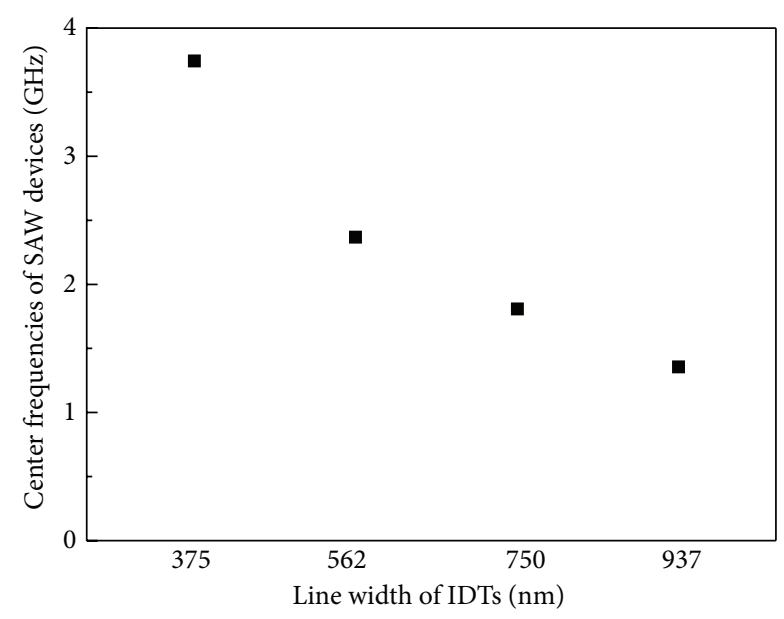

Figure 12: The center frequency of SAW devices with various line widths of IDTs.

implemented with a Rayleigh-mode at $1.36 \mathrm{GHz}, 1.81 \mathrm{GHz}$, $2.37 \mathrm{GHz}$, and $3.74 \mathrm{GHz}$ are successfully fabricated in this study. Results of this study demonstrate that the proposed high-frequency SAW device is highly promising for use in $4 \mathrm{G}$ mobile telecommunication.

\section{Conflict of Interests}

The authors declare that there is no conflict of interests regarding the publication of this paper.

\section{Acknowledgments}

The authors would like to thank the National Science Council, Taiwan, for financially supporting this research under Contract nos. NSC102-2221-E-366-002, NSC102-2221-E-110-029, and NSC102-2221-E-237-005 and Aim for the Top University Project.

\section{References}

[1] A. Pocovnicu, "Biometric security for cell phones," Informatica Economică, vol. 13, pp. 57-63, 2009.

[2] R. C. Ruby, P. Bradley, Y. Oshmyansky, A. Chien, and J. D. Larson III, "Thin film bulk wave acoustic resonators (FBAR) for wireless applications," in Proceedings of the IEEE Ultrasonics Symposium (IUS '01), vol. 1, pp. 813-821, Atlanta, Ga, USA, October 2001.

[3] J. D. Larson III, R. C. Ruby, P. D. Bradley, J. Wen, S.-L. Kok, and A. Chien, "Power handling and temperature coefficient studies in FBAR duplexers for the $1900 \mathrm{MHz}$ PCS band," in Proceedings of the IEEE Ultasonics Symposium (IUS '00), vol. 1, pp. 869-874, San Juan, Puerto Rico, USA, October 2000.

[4] K. M. Lakin, K. T. McCarron, J. Belsick, and R. Rose, "Filter banks implemented with integrated thin film resonators," in Proceedings of the IEEE Ultasonics Symposium (IUS '00), vol. 1, pp. 851-854, San Juan, Puerto Rico, USA, October 2000.

[5] R. Ruby, "Review and comparison of bulk acoustic wave FBAR, SMR technology," in Proceedings of the IEEE Ultrasonics
Symposium (IUS ’07), pp. 1029-1040, New York, NY, USA, October 2007.

[6] Q.-X. Su, P. Kirby, E. Komuro, M. Imura, Q. Zhang, and R. Whatmore, "Thin-film bulk acoustic resonators and filters using $\mathrm{ZnO}$ and lead-zirconium-titanate thin films," IEEE Transactions on Microwave Theory and Techniques, vol. 49, no. 4, pp. 769-778, 2001.

[7] S.-Y. Chu, T.-Y. Chen, I.-T. Tsai, and W. Water, "Doping effects of $\mathrm{Nb}$ additives on the piezoelectric and dielectric properties of PZT ceramics and its application on SAW device," Sensors and Actuators A: Physical, vol. 113, no. 2, pp. 198-203, 2004.

[8] A. Umar, M. M. Rahman, M. Vaseem, and Y.-B. Hahn, "Ultrasensitive cholesterol biosensor based on low-temperature grown $\mathrm{ZnO}$ nanoparticles," Electrochemistry Communications, vol. 11, no. 1, pp. 118-121, 2009.

[9] R.-C. Lin, K.-S. Kao, C.-C. Cheng, and Y.-C. Chen, "Deposition and structural properties of RF magnetron-sputtered $\mathrm{ZnO}$ thin films on $\mathrm{Pt} / \mathrm{Ti} / \mathrm{SiN}_{x} / \mathrm{Si}$ substrate for FBAR device," Thin Solid Films, vol. 516, no. 16, pp. 5262-5265, 2008.

[10] R.-C. Lin, Y.-C. Chen, and K.-S. Kao, “Two-step sputtered ZnO piezoelectric films for film bulk acoustic resonators," Applied Physics A: Materials Science and Processing, vol. 89, no. 2, pp. 475-479, 2007.

[11] C.-J. Chung, Y.-C. Chen, C.-C. Cheng, and K.-S. Kao, "An improvement of tilted AlN for shear and longitudinal acoustic wave," Applied Physics A: Materials Science and Processing, vol. 94, no. 2, pp. 307-313, 2009.

[12] C.-L. Wei, Y.-C. Chen, S.-R. Li, C.-C. Cheng, K.-S. Kao, and C.-J. Chung, "Effects of reflecting layers on resonance characteristics of a solidly mounted resonator with $1 / 4 \lambda$ mode configuration," Applied Physics A: Materials Science and Processing, vol. 99, no. 1, pp. 271-278, 2010.

[13] S. Hong, J. Yeo, W. Manorotkul et al., "Low-temperature rapid fabrication of $\mathrm{ZnO}$ nanowire UV sensor array by laser-induced local hydrothermal growth," Journal of Nanomaterials, vol. 2013, Article ID 246328, 7 pages, 2013.

[14] G. Zhang, "Nanostructure-enhanced surface acoustic waves biosensor and its computational modeling," Journal of Sensors, vol. 2009, Article ID 215085, 11 pages, 2009.

[15] Y. C. Chen, W. T. Chang, K. S. Kao, C. H. Yang, and C. C. Cheng, "The liquid sensor using thin film bulk acoustic resonator with C-axis tilted AlN films," Journal of Nanomaterials, vol. 2013, Article ID 245095, 8 pages, 2013.

[16] J. G. Rodríguez-Madrid, G. F. Iriaróte, J. Pedros, O. A. Williams, D. Brink, and F. Calle, "Super-high-frequency SAW resonators on AlN/diamond," IEEE Electron Device Letters, vol. 33, no. 4, pp. 495-497, 2012.

[17] M. B. Assouar, O. Elmazria, P. Kirsch, P. Alnot, V. Mortet, and C. Tiusan, "High-frequency surface acoustic wave devices based on AlN/diamond layered structure realized using e-beam lithography," Journal of Applied Physics, vol. 101, no. 11, Article ID 114507, 2007.

[18] P. Kirsch, M. B. Assouar, O. Elmazria, V. Mortet, and P. Alnot, " $5 \mathrm{GHz}$ surface acoustic wave devices based on aluminum nitride/diamond layered structure realized using electron beam lithography," Applied Physics Letters, vol. 88, no. 22, Article ID 223504, 2006.

[19] Y. Takagaki, P. V. Santos, E. Wiebicke, O. Brandt, H.-P. Schönherr, and K. H. Ploog, "Superhigh-frequency surfaceacoustic-wave transducers using AlN layers grown on $\mathrm{SiC}$ substrates," Applied Physics Letters, vol. 81, no. 14, pp. 25382540, 2002. 
[20] P.-T. Hsieh, H.-S. Chin, P.-K. Chang, C.-M. Wang, Y.-C. Chen, and M.-P. Houng, "Effects of the annealing environment on green luminescence of $\mathrm{ZnO}$ thin films," Physica B: Condensed Matter, vol. 405, no. 11, pp. 2526-2529, 2010.

[21] W.-T. Chang, Y.-C. Chen, R.-C. Lin, C.-C. Cheng, K.-S. Kao, and Y.-C. Huang, "Wind-power generators based on $\mathrm{ZnO}$ piezoelectric thin films on stainless steel substrates," Current Applied Physics, vol. 11, no. 1, pp. S333-S338, 2011.

[22] G. S. May, J. Huang, and C. J. Spanos, "Statistical experimental design in plasma etch modeling," IEEE Transactions on Semiconductor Manufacturing, vol. 4, no. 2, pp. 83-98, 1991. 

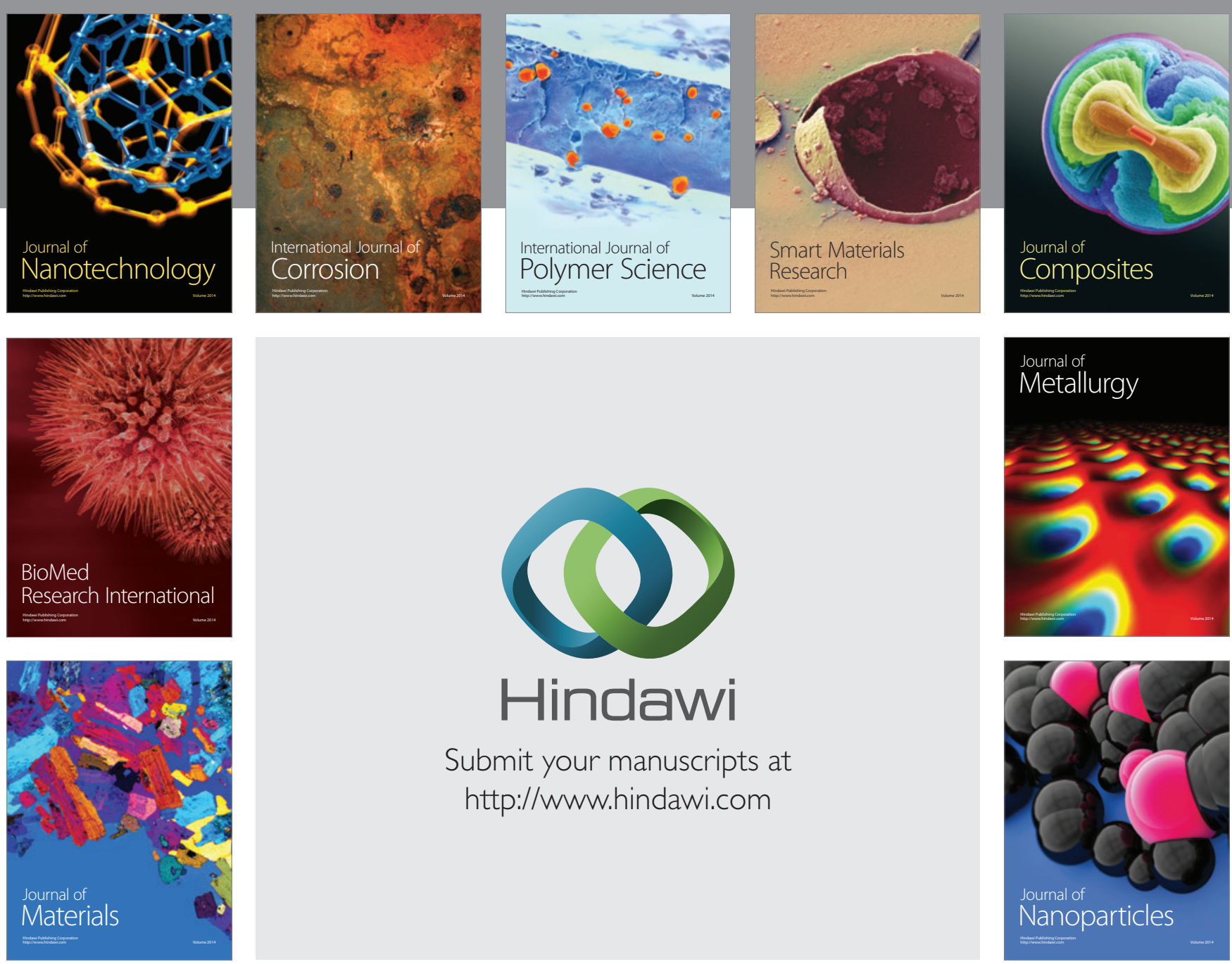

Submit your manuscripts at http://www.hindawi.com
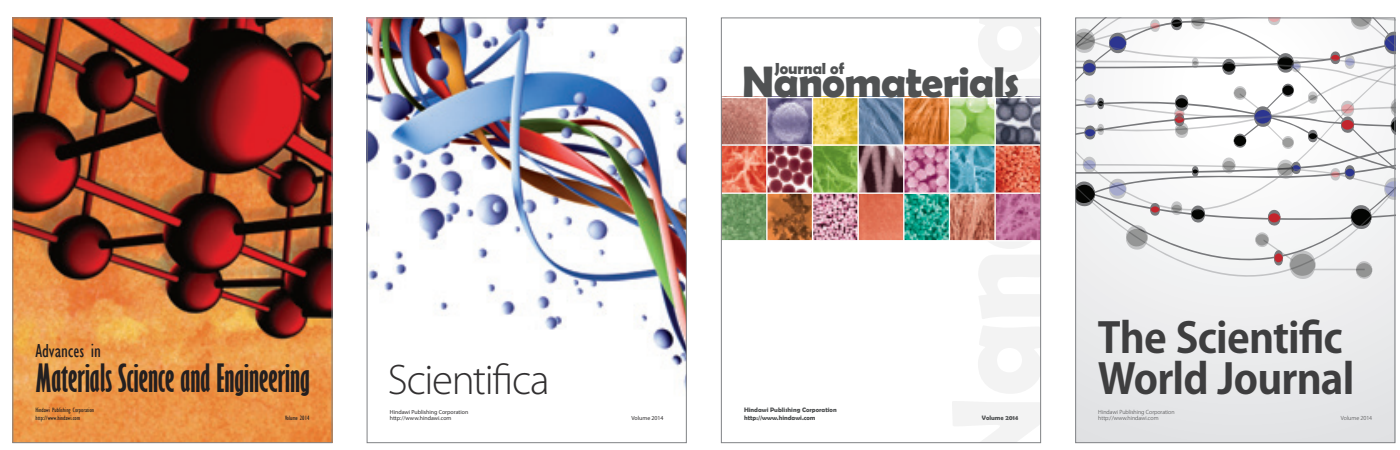

\section{The Scientific World Journal}
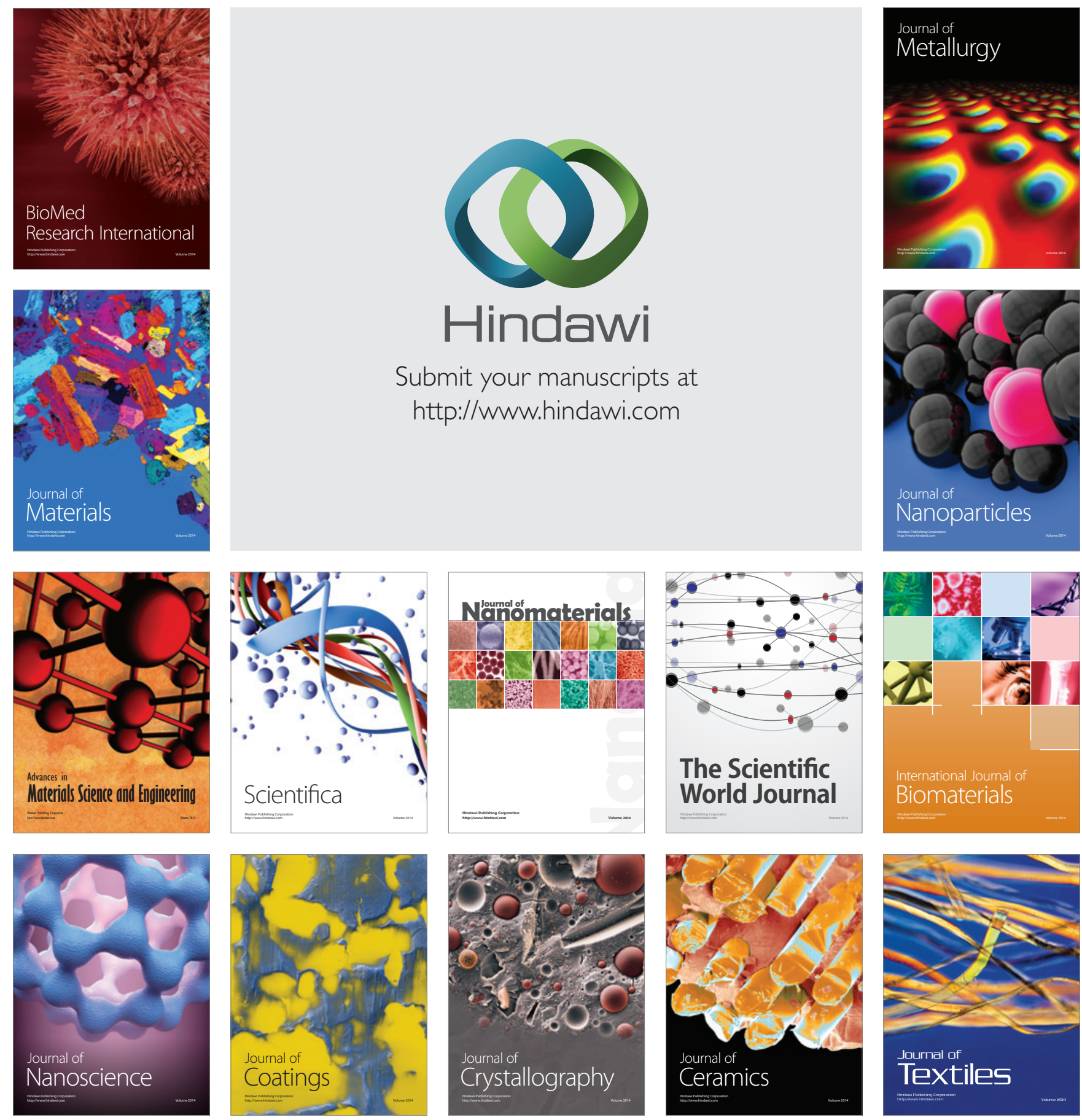